\title{
Hardware/Software Co-design of Physical Unclonable Function based Authentications on FPGAs
}

\author{
Aydin Aysu*, Patrick Schaumont \\ Bradley Department of ECE, Virginia Tech, Blacksburg, VA 24061, USA
}

\begin{abstract}
Physical Unclonable Functions (PUFs) enable the generation of device-unique, on-chip, and digital identifiers by exploiting the manufacturing process variation. The past decade has seen an extensive effort in PUF design. Yet, most PUF constructions are regarded as stand-alone hardware building blocks. In contrast, we propose PUF constructions that are tightly integrated into the design of a micro-processor. The proposed PUFs are essentially a collection of time-to-digital converters that are integrated into the custom instruction or memory-mapped interface of a processor. Therefore, the processor can issue the PUF challenges and collect the associated responses using instruction executions. This integration enables practical, run-time physical authentication and it allows flexible post-processing mechanisms using software. In this article, we describe the design, implementation, and the performance analysis details of such hardware/software co-designed authentication mechanisms on FPGAs. We propose two variants of the PUF architecture: a synchronous module that requires minimal place and route constraints utilizing the common clock of the SoC, and an asynchronous alternative that is independent of the clock but realized with a controlled placement. We implemented the synchronous architecture on the Altera Cyclone-IV FPGAs and performed a large-scale characterization on 55 boards. The asynchronous design is realized on the Xilinx Virtex-5 FPGAs and tested on 100 boards. Measurements reveal that the proposed solutions can authenticate trillions of devices and provide better performance than the ring oscillator based alternative.
\end{abstract}

(C) 2014 Published by Elsevier Ltd.

Keywords: Physical Uncloneable Functions, System-on-Chip Integration, HW/SW Co-design, Ring Oscillator, Time-to-digital converters, FPGA

\section{Introduction}

Physical Unclonable Functions (PUFs) exploit the random process variations of the manufacturing process for establishing device-unique electronic signatures. PUFs are defined by challenge/response pairs where a challenge is a distinct input and a triggering method of the PUF, and a response is the generated output. The device-unique unclonable output responses can serve a wide variety of applications such as anti-counterfeiting

\footnotetext{
Corresponding author

Email address: aydinay@vt.edu (Aydin Aysu)
}

[1], tamper-resistant key storage [2], bitstream protection [3], and IP binding [4].

We propose a PUF alternative that utilizes the critical timing violation frequency $(1 / \Delta t)$ of data paths. These values can be exploited as a device-unique feature [5], [6]. Figure 1 illustrates a generic structure of such PUFs. First, R1 and R2 are initialized to a known state. Then, at time 0 , the input register launches the new value from R1. This value propagates through the data path and after a device-dependent, finite amount of time $(\Delta t)$, we can capture the expected value at $\mathrm{R} 2$. In order to estimate the value of $\Delta t$, we can use a mechanism that sweeps the frequency of the clock input of 


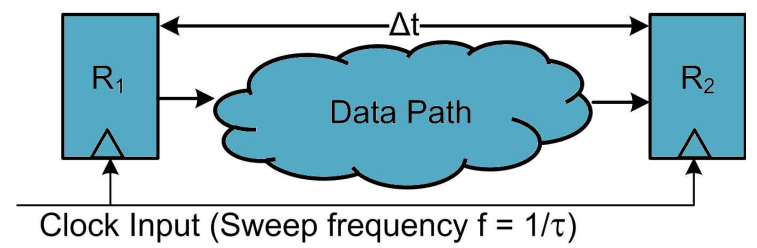

Figure 1. Critical timing $(\Delta t)$ is the time required to generate the value at R2, that starts from the output of R1 and passes through the data path. A clock period of $\tau<\Delta t$ causes a violation of the critical timing.

both R1 and R2, and check the correctness of the value at R2 [5]. However, clock sweeping is an expensive process that often requires an external clock generator circuitry or reconfigurable PLLs.

Figure 2 shows a generic method of dodging clock sweeping and still obtaining a device-unique value. Instead of using a single data path, we could use multiple paths which have distinct $\left(\Delta t_{i}\right)$ values. If we can set the critical timing of the paths in an ascending order $\left(\Delta t_{1}<\Delta t_{2}<\Delta t_{3}<\ldots<\Delta t_{n}\right)$, we can fix the clock frequency, and instead check starting at which data path, executions start to fail. This data path may vary on different devices. We have built our PUF based on this key insight. We essentially exchange the requirement of the clock sweeping with a custom instruction integrated into a processor, enabling critical timing violations at a nominal and fixed clock frequency.

The major contributions of this work are:

- To propose alternative PUF architectures on Xilinx and Altera FPGAs that are able to generate device-unique identifiers at run-time without a clock sweeping mechanism.

- To present a complete SoC platform that can perform run-time authentication with the proposed PUF architecture. The PUF is integrated into the processor using a custom instruction (for Altera) or memory mapped interface (for Xilinx). The extraction of the authentication identifiers, with all the required post-processing can be performed in software. The SoC platform achieves authentication by combining the flexibility of the software with the static entropy of the hardware.

- To show the detailed results of a large scale characterization on 55 Altera and 100 Xilinx FPGAs with comparable previous designs, and to estimate the performance of the authentication identifiers.

The rest of the paper is organized as follows. Section 2 gives a brief overview of the previous work on FPGA

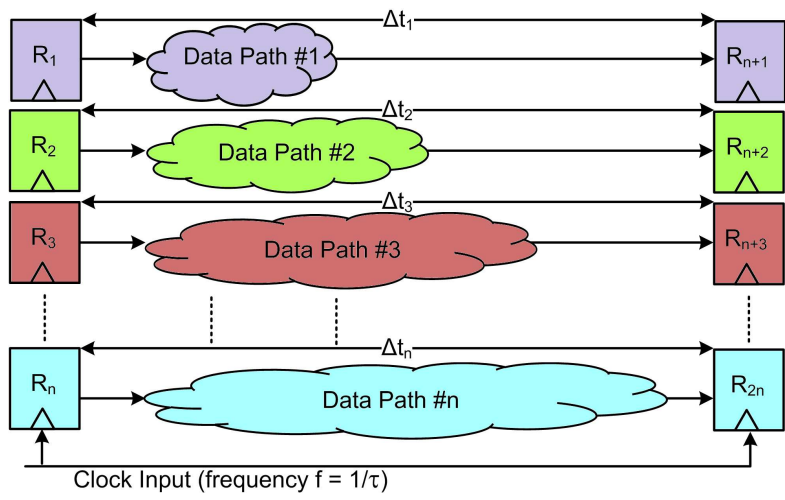

Figure 2. Generic structure of a PUF that can utilize critical timing violations using a fixed clock source.

based PUFs and motivates our design. Section 3 analyzes the principle of operation and compares it with the construction of Maiti et al. [5]. Section 4 presents our PUF, its integration to the SoC architecture, and some SW-based post-processing methods to generate authentication identifiers out of raw PUF responses. Section 5 proposes an asynchronous PUF variant for Xilinx FPGAs. Section 6 quantifies the performance of authentication identifiers for the proposed solutions and compares it with ring oscillator based PUF constructions. Section 7 concludes the paper.

\section{Previous Work and Motivation}

There have been many PUF constructions on various platforms: refer to Maes et al. for an overview on PUF proposals [15]. Figure 3 shows the universal set of PUFs and the subset of interest. In this paper, we focus on the relaxed FPGA based PUF implementation as the number of viable solutions are limited. This family of PUFs can be implemented with relaxed place and routing constraints (eg. using Altera logiclock) and do not require bitstream manipulations.

\subsection{A Brief Overview of FPGA based PUFs}

A popular choice for PUF constructions is to use memory elements. These PUFs typically exploit the transistor mismatches of SRAM cells. Maes et al. propose a method to port a memory based PUF to FPGAs [10]. They use the initial power-up values of the registers. Depending on the process variations, when the power is turned on, the value of a flip-flop can be either 0 or 1 which can be used as a device-unique signature. Kumar et al. presents the Latch PUF that utilize crosscoupled latches [12]. Latches are set to '1' and reset 


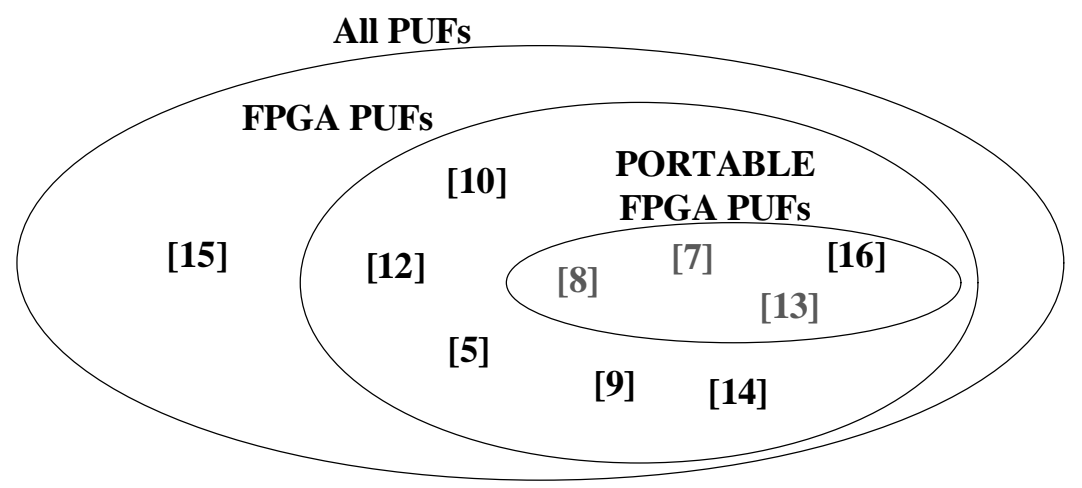

Figure 3. The universal set of PUF and the subsets of interest. [7], [8], and [9], are colored in gray because their placement and routing constraints can be relaxed but this causes a drastic decrease in their performance.

Table 1. FPGA PUF Constructions and Requirements

\begin{tabular}{c|c|c|c}
$\begin{array}{c}\text { Resource of } \\
\text { Variation }\end{array}$ & $\begin{array}{c}\text { Measured } \\
\text { Variation }\end{array}$ & $\begin{array}{c}\text { Technology } \\
\text { Requirement }\end{array}$ & Reference \\
\hline Memory & $\begin{array}{c}\text { Power-up } \\
\text { Values, } \\
\text { Write Collisions }\end{array}$ & $\begin{array}{c}\text { Power cycling, } \\
\text { Bitstream Manipulation, } \\
\text { True Dual-Port SRAMs }\end{array}$ & [10], [11] [12] \\
\hline $\begin{array}{c}\text { Delay } \\
\text { Elements }\end{array}$ & $\begin{array}{c}\text { Timing of Delay } \\
\text { Paths }\end{array}$ & $\begin{array}{c}\text { Strict Control over } \\
\text { Placement and Routing }\end{array}$ & [7], [8], [9], [13], [14] \\
\hline Microprocessor & $\begin{array}{c}\text { Ciritical Timing } \\
\text { Violation } \\
\text { Frequency }\end{array}$ & $\begin{array}{c}\text { External High- } \\
\text { Precision Clock } \\
\text { Generator }\end{array}$ & {$[5]$} \\
\hline $\begin{array}{c}\text { Custom } \\
\text { Instruction }\end{array}$ & $\begin{array}{c}\text { Critical Timing } \\
\text { Violation Path }\end{array}$ & $\begin{array}{c}\text { Custom Instruction } \\
\text { Execution }\end{array}$ & This work
\end{tabular}

to ' 0 ' at the same time, and do ultimately converge to one value based on intrinsic variation. Finally, Güneysu proposes an alternative that exploits memory-write collisions [11].

An alternative PUF group is the ones that PUFs exploit delay variations. We can divide this group further into arbiter, oscillator, and glitch based PUFs. Arbiter PUFs on FPGAs are presented in [7], [8]. The principle of Arbiter PUF is to race two paths with the same intended delay and select the faster one. Even if we design two paths with the same delay, process variation causes deviations from the intended values, and randomly but statically, makes one to be slightly faster. Anderson provides the glitch PUF as an alternative for delay based PUFs [9], which exploits the variability of the length of an artificially generated glitch. Oscillator based constructions offer another solution [13], [16]. If we invert the output of a path and feed it back to its input, we can create a ring oscillator ( $\mathrm{RO})$. The oscillation frequency of ROs differ due to the process variation and we can use it as a PUF.

\subsection{Motivation of this work}

Given the number of available PUF solutions, why do we need yet another one? Most of the current PUF proposals are deeply embedded into the FPGA fabric, and require a tight control over the logic placement or the power switches. In contrast, our solution needs relatively less backend-specific requirements and can be ported to platforms (eg. Altera FPGAs) where the designer is not able to handpick logic elements at the lowest level.

Table 1 summarizes the PUF constructions on FPGAs. Memory based PUFs require bitstream manipulation, power cycling or true-dual port SRAMs. Most of the delay based PUFs and the latch PUF use some form of race or comparison mechanism that need fine tuning. Therefore, the quality of these PUFs reduces drastically if the control over the placement and routing tools is limited (eg. Altera FPGAs). Cherif et al. proposes some tricks on the RO-PUF to reduce this effect on Altera FPGAs [16] and we compare our results with them in the result section.

The PUF presented by Maiti et al. can be regarded as 


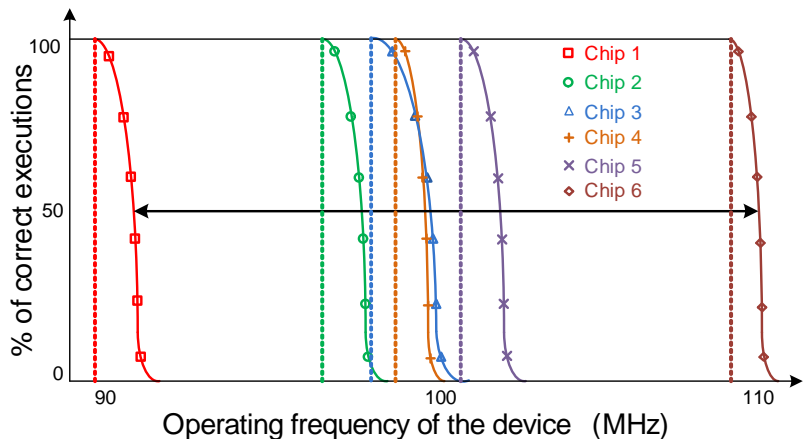

(a)

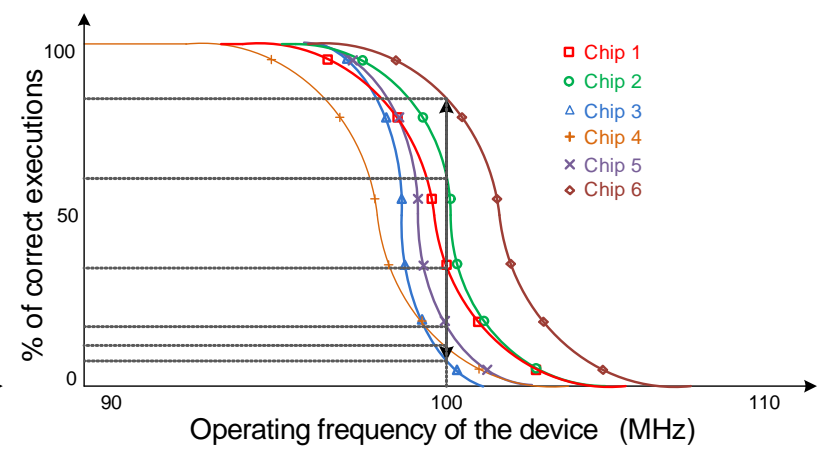

(b)

Figure 4. The principle of operation for PUF construction in [5] (a) and the one proposed in this work (b). In [5], the input clock frequency is swept and the chip identity is measured as a function of frequency, whereas the proposed construction uses a fixed clock input and the chip identity is measured as a function of percentage of correct executions.

a viable alternative to delay based PUFs, but it requires a sweeping mechanism using an external clock source to generate a critical timing violation [5]. Therefore, it is not suitable for run-time authentication on low-cost devices that have a fixed and stable clock source. Moreover, it can only be used if the store instruction is not on the critical path. However, the proposed PUF does not use such a method, it observes the critical path violation with a fixed clock frequency and allows implementation with a relaxed place and route constraints.

Moreover, there is also a lack of prototype test platforms for physical authentication on reconfigurable devices. In this paper, we propose such a complete SoC platform with novel PUF architectures that do not require a varying clock source and that offer a seamless integration to the architecture of the test platform. The proposed PUFs are integrated into the custom instruction interface of the NIOS-II processor or the memory mapped interface of the MSP430 processor. Therefore, PUF responses can be issued by instruction calls which allow run-time authentication and enable implementation of post-processing in software.

\section{Principle of Operation}

Figure 4(a) depicts the core idea of Maiti's PUF construction [3]. The $\mathrm{X}$ axis of this figure shows the input clock frequency, and the $\mathrm{Y}$ axis shows the percentage of correct executions. On each device, process variation causes small differences to the critical timing of the instruction executions. These differences are measured by a mechanism that sweeps the frequency range of the clock input and and that checks the correctness of the execution at different clock frequencies. Then, these execution time variations are utilized as device-unique identifiers. There are two problems with the use of this method on a processor with a fixed-frequency clock: (i) The variation between the \% of correct executions are too wide and (ii) it drops down rapidly from $100 \%$ to $0 \%$. Therefore, on a fixed clock frequency, most of the chips will have the same violation behavior. For example, if we select $100 \mathrm{MHz}$ as the fixed frequency of the input clock in Fig. 3 (a), we could separate Chip3 and Chip4 since they would have different error ratio. However, we would not be able to differentiate Chip1 from Chip2, and Chip5 from Chip6 since they would have exactly the same error behavior.

Figure 4 (b) gives the principle of operation for the proposed stable-clocked PUF. Our construction uses a fixed input clock with a carefully designed custom instruction. By design, the custom instruction drops down slowly from $100 \%$ correct executions to $0 \%$ correct executions. In contrast to the behavior in figure 4(a), the chips that use our principle of operation in figure 4(b) would have different \% of correct executions at the fixed and the nominal clock frequency of $100 \mathrm{MHz}$.

\section{Physical Authentication}

\subsection{Novel PUF}

We propose a non-trivial combination of PUF and software that can only be achieved by a tight systemlevel integration. In this section, we first describe the 


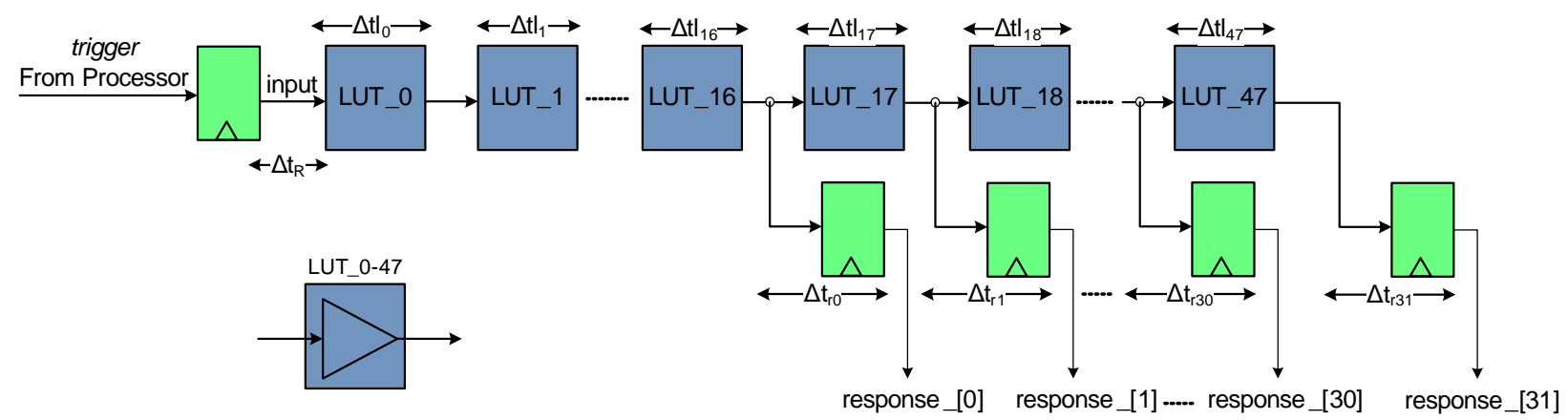

Figure 5. Architecture of a proposed PUF block. Multiple copies of these blocks are used to generate the complete device-unique identifier.

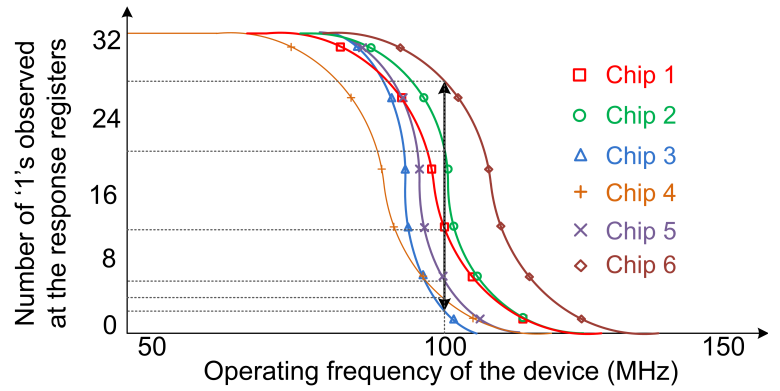

Figure 6. Number of $1 \mathrm{~s}$ at the output registers vs. operating frequency of the processor.

synchronous PUF construction, then we explain its system integration, and finally propose the applied software post-processing to generate the device-unique identifiers.

Figure 5 gives the details of the proposed PUF block. The PUF block is a time-to-digital converter. We configure LUT_0 to LUT_47 as buffers composing a delay-line and capture the output of LUT_16 to LUT_47 in a register. The output of these 32 registers generate a 32-bit output response that can be returned to the processor efficiently using the custom instruction interface. In order to pool a rich source of entropy, the complete PUF architecture consists of multiple copies of the PUF block from Figure 5.

The proposed PUF architecture works as follows. The processor uses the custom instruction interface to send the 1-bit input trigger to the PUF block. It also generates a start signal to synchronize the input. The processor enables the input-register when the start signal is ' 1 ' and capture the value of the 1-bit input trigger. Then, at the next clock cycle, the hardware evaluates the PUF; it propagates the input signal through the delay-line and stores it at the registers. Depending on the clock frequency, a subset of these registers will be updated to ' 1 '. Next, we derive a formal definition for these operations.

It takes a finite amount of time to complete the required operations: $(i)$ to read out the value of a register $\left(\Delta t_{R}>0\right)$, (ii) to propagate it through the delay-line $\left(\Delta t l_{i}>0\right)$, and (iii) to write it into a register $\left(\Delta t r_{i}>0\right)$. Previous work [5], [6] showed that these values $\left(\Delta t_{R}\right.$, $\left.\Delta t l_{i}, \Delta t r_{i}\right)$ are device-unique random values. If the clock period is shorter than the time required to complete this path, a critical timing violation occurs. Equation 1 formulates a condition for the critical timing violation where $\tau$ stands for the clock period. If equation 1 is satisfied, the value of at least one output register does not get updated.

$$
\Delta t_{R}+\sum_{i=0}^{47} \Delta t l_{i}+\sum_{i=0}^{31} \Delta t r_{i}<\tau
$$

We set the number of LUTs that generates the delayline so that $\tau$ satisfies the conditions of 2 and 3. As an example, the delay-line in figure 5 use 48 buffers (LUT_0 to LUT_47).

$$
\begin{aligned}
& \Delta t_{R}+\sum_{i=0}^{n} \Delta t l_{i}+\Delta t_{r n}<\tau \\
& \Delta t_{R}+\sum_{i=0}^{n+1} \Delta t l_{i}+\Delta t_{r(n+1)}>\tau
\end{aligned}
$$

If equation 2 and 3 are both satisfied, then the value of the $n$-th register will get updated but the value of $(n+1)$ th register will remain the same. Since $\Delta t_{R}, \Delta t l_{i}, \Delta t r_{i}$ are device-unique random values, then the value of $n$, which is a linear combination of these random values, will also be random. 


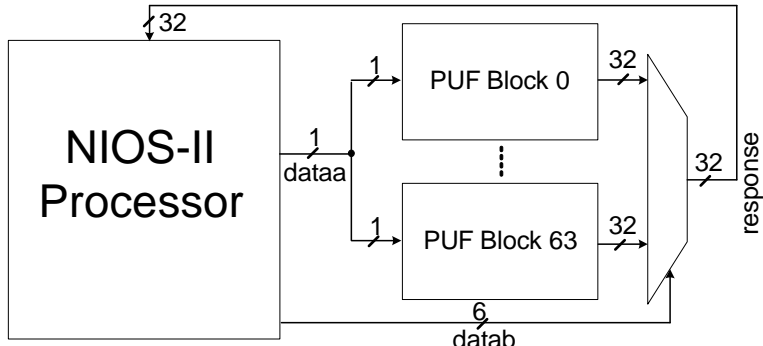

Figure 7. High-level PUF block diagram.

Following the randomness observation, we introduce a method to formalize the challenge response pairs and the reasoning behind this construction. During inactive mode (when the PUF is not used), all registers are preset to '0'. The processor issues a challenge 1bit trigger input that is set to ' 1 '. Using the delayline, the PUF block generate a timing violation on the output registers and utilize the random variations of $\Delta t_{s}, \Delta t l_{i}, \Delta t r_{i}$. Our experiments showed that $\Delta t r_{i}<$ $\Delta t l_{i}+\Delta t r_{(i+1)}$ (for $\left.i=0,1, \ldots, 31\right)$. Hence, the time it takes to write into a register $(n)$ is less than the time it takes to write into the next register $(n+1)$. Therefore, on the 32-bit output register, the resulting value will be a string of ' 1 's followed by a string of '0's (eg $11111111110000000000000000000000)$. Since this is the result of a device-unique process variation, it can be utilized as static randomness. Concurrently, PUF also generates an output done signal to indicate to the processor that it has generated a response.

Figure 6 shows the realization of our principle of operation where $\mathrm{X}$ axis represents the operating frequency of the chip and the $\mathrm{Y}$ axis is the number of $1 \mathrm{~s}$ observed at the output registers. If the frequency is low (the clock period $\tau$ is high), the value ' 1 ' of the input can propagate through the line and captured at all the output registers as there are no timing violations. Hence, the value of the output registers are all '1's. On the opposite end, if the operating clock frequency is very high (the clock period $\tau$ is low), the next clock edge may come even before the input can reach to the first register. This will cause all registers to stay at their preset value of 0 . If the clock frequency is in the middle of these extreme ends (eg. $100 \mathrm{MHz})$, then due to process variation, the output is different on different devices. Therefore, the proposed realization can use a fixed clock frequency and can still utilize timing violations as device-unique identifiers on FGPAs.

Figure 7 shows the high-level block diagram of the complete PUF architecture. The prototype architecture has 64 copies of this PUF block. The processor selects

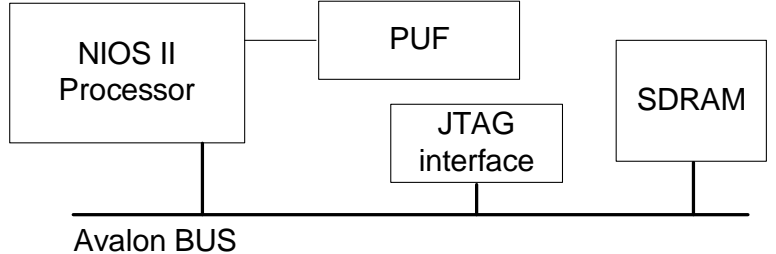

Figure 8. The high-level architecture of the Altera prototype.

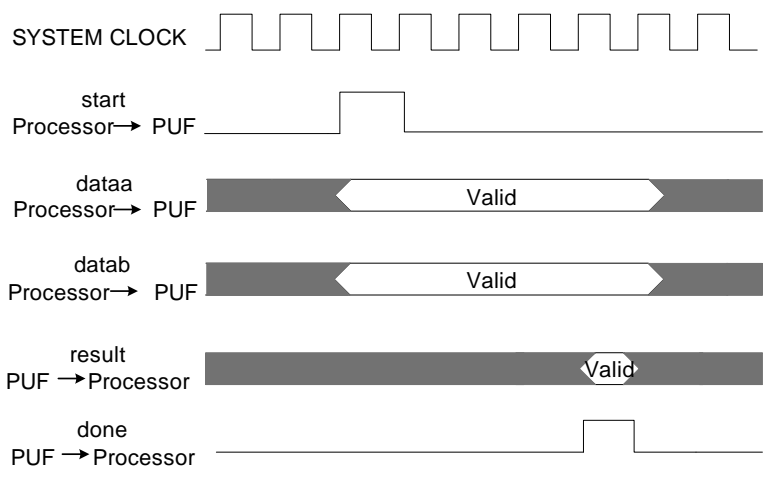

Figure 9. Timing diagram of the communication between processor and PUF using the custom instruction interface.

the final 32-bit response out of these multiplexed PUF blocks. The processor performs this selection by using the input signal datab of the custom instruction interface. For $n$-copies of the PUF block, the total number of raw responses is also $n$. Depending on the entropy requirement of the application, a designer can scale the number of total PUF blocks. More PUF blocks generate more responses and enable more entropy for deviceunique identifiers and the section 6 elaborates further on the required number of PUF blocks. The current prototype can easily support a large number of PUF blocks since the datab width scales logarithmically.

\subsection{System Integration}

Figure 8 shows the high-level architecture of our prototype. The system consists of a NIOS-II softcore processor, a 32-Kb SRAM, a JTAG interface for programming, Avalon switch fabric for communication with the embedded peripherals, and the proposed PUF architecture for physical authentication. We integrate the proposed PUF into the NIOS-II softcore processor via a custom instruction interface. The PUF responses can be requested by an instruction using a $C$ code as in equation 4 . When the software calls the function

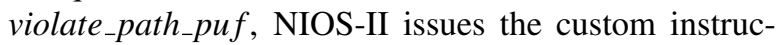
tion for PUF where dataa and datab are the inputs of this function and the return value response is the output. 


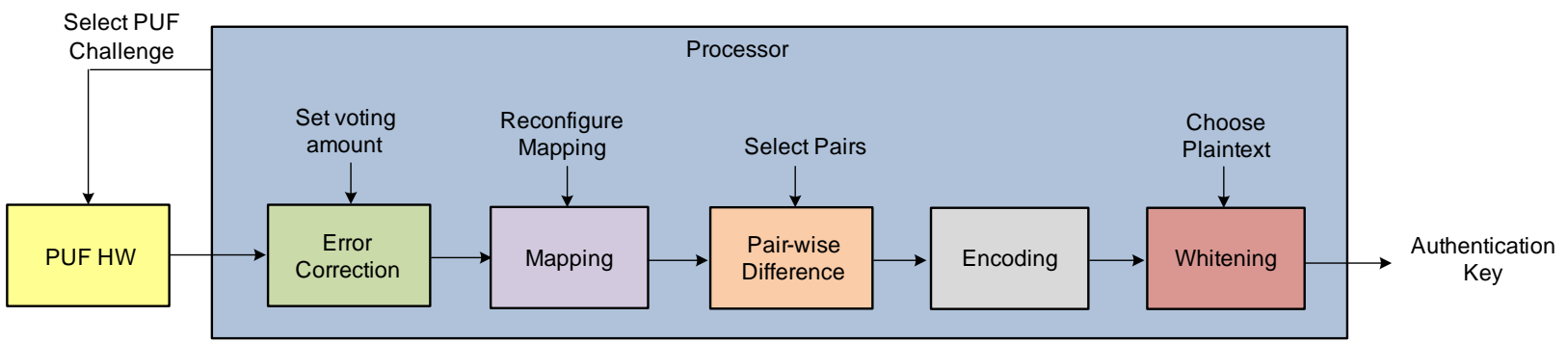

Figure 10. Block diagram of PUF hardware, and software post-processing

All dataa, datab and response variables are defined as 32-bit integers.

$$
\text { response }=\text { violate_path_puf(dataa, datab); }
$$

We illustrate the timing diagram of the custom instruction interface in figure 9 [17]. When the processor issues a PUF instruction, the start signal becomes ' 1 ', for one clock cycle. At the same clock cycle, the values of dataa and datab are sampled by the custom instruction. These data inputs remain valid until the PUF instruction returns the result. When PUF-output response is available, a done signal is generated for one clock cycle, the processor reads out the value result, and stores it in response. The processor remains idle during this custom instruction execution.

\subsection{Generating Authentication Keys From Raw PUF Responses}

Since the processor issues the PUF instruction and the response is returned back to it, software can perform several post-processing to improve the quality of the responses. Rather than proposing the best possible post-processing methods, this work shows that the proposed prototype enables flexible software postprocessing mechanisms that can possibly increase the reliability, uniqueness and the entropy of the raw device-unique identifiers.

Figure 10 shows the flow-graph of the authentication identifier generation. We utilize five basic operations; Majority Voting, Mapping, Pair-wise Difference, Encoding, and Whitening. The first step is to apply a Majority Voting on the raw PUF responses, which is fundamentally applying the same challenge a number of times and choosing the most occurring response. This process filters out low-frequency noise and is repeated one by one for each PUF block. The user has the flexibility to dynamically configure the number of issued votes and can trade-off reliability for execution time. The reliability can also be further enhanced by implementing an error correcting code like the $\mathrm{BCH}$ codes.

After the Majority Voting, software performs Mapping which is the step where the digital responses are mapped into arithmetic values. For the proposed designs, the PUF HW output ranges between '0x00000000' and '0xFFFFFFFF' and is mapped from ' 0 ' to ' +32 ' accordingly. Software enables the adjustment of this mapping for different FPGA device families.

The next step is to perform the Pair-wise Difference. This process is typically used to reduce systematic variations and to decrease the temporal noise [18]. Instead of using the absolute responses, which may change due to bias effects and operating conditions (eg. voltage drops and temperature), pairing responses and using their relative relation is more susceptible to noise. For $n$ PUF blocks in the architecture, the total number of pair-wise differences are $(n \times n-1) / 2$, with $\log 2(n !)$ bits of entropy [19]. Software enables a designer to use a subset of these pairs that are more reliable and/or unique. To minimize the systematic variations and simplify the selection process, our system compares the adjacent blocks (eg. block(0-1),block(1-2), block(2-3), etc.).

Following the Pair-wise Difference, an Encoding scheme returns the resulting arithmetic values into 1-bit encoded symbols. This operation increases the entropy since it maps multiple bits into single bit variables. The default assignment for the Encoding is ' 0 ' for the most occurring response and ' 1 ' for all others.

Finally, the Whitening process further improves the uniqueness of the identifier. To implement this operation, the proposed platform uses a 128-bit Advanced Encryption Standard (AES) block cipher. The output of the pair-wise difference is the key of this cipher and the software can generate a ciphertext challenge. Therefore, this would result in a large space of $2^{128}$ challengeresponse pairs. Depending on the application require- 


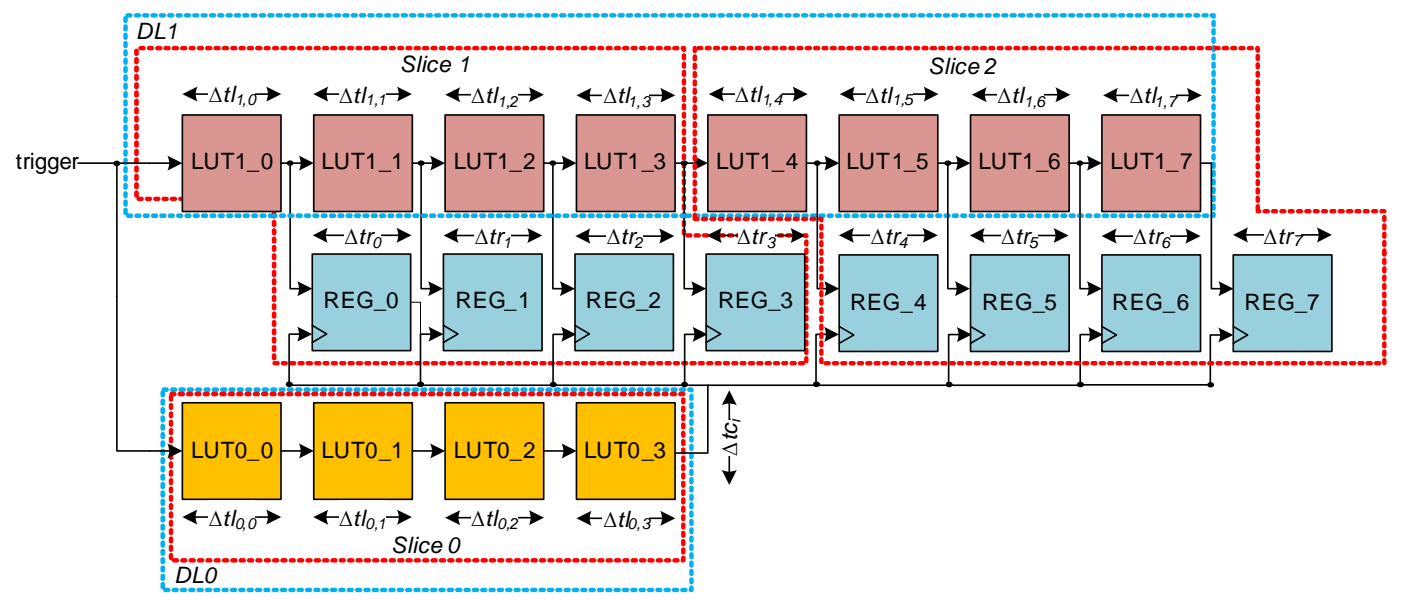

Figure 11. The hardware architecture of the asynchronous PUF

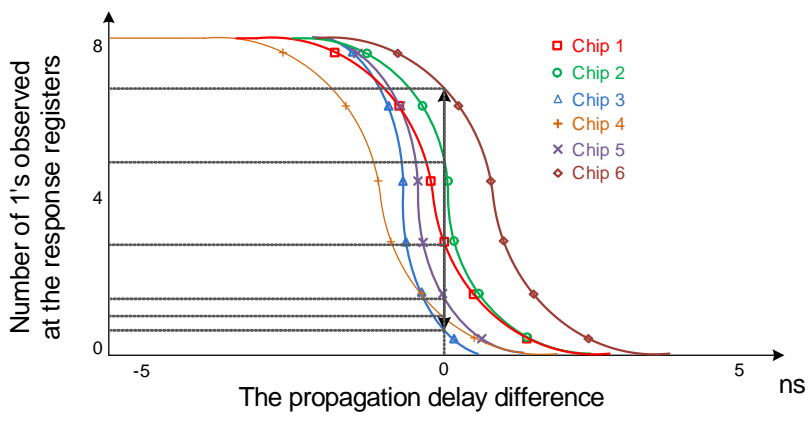

Figure 12. The number of '1's at the output registers vs. the timing differences of the delay-lines

ments the software can opt for enabling/disabling the whitening. This feature should be used with care as it will expand a single-bit error on its input into multiple bits.

\section{An Asynchronous violation-based PUF variant}

The major drawback of the PUF architecture proposed in section 4.1 is its dependence to the clock input. If the clock of the system is not within a specified bound or if the design is migrated to a substantially different FPGA, the output may not reveal a PUF characteristic. Therefore, we propose a PUF variant that utilizes an asynchronous design to mitigate this issue. This version is exclusively implemented on the Xilinx FPGA architecture as it provides a more detailed control over the placement and routing tools.

The main idea behind this construction is to compare the delay disparity of two delay-lines. The key difference from the previous solution is that this time, rather than extracting the timing violations with respect to a global clock source, we utilize an asynchronous approach. Yet, the fundamental principles of timing violation PUF applies to this architecture as well. Figure 11 highlights the hardware architecture of the asynchronous PUF. The design consists of two delay-lines: DL0 and DL1. DL0 has $n$ delay elements (LUTs) while DL1 has $2 n$ elements and registers to capture the output of the delay-line. These registers get its data input from DL1 and its clock from DL0. Initially the registers are set to '0'. Then, the processor enables the registers for a PUF evaluation. When enabled, a register will only set its logical value to ' 1 ' if it receives the clock edge before the data input. Therefore, if the architecture utilizes $n$ delay elements, it will measure how further the DL1 propagates (within the range 0 to $2 n$ ) until DL0 propagates by $n$ steps. The input trigger of the PUF block can be the system clock but the design is asynchronous in the sense that PUF generates the clock input of its output registers.

The formulation of the PUF block is as follows. The time it takes for a signal to propagate through a delay element is $\Delta t l_{u, v}$, where $u$ is the index of the DL and $v$ is the index of the LUT. The time it takes for a signal to be properly written into a register is again corresponds to $\Delta t r_{i}$ and the routing delay of the clock input of a register is $t c_{i}$ where $i$ is the index of the register. In figure 11, the clock input of all registers passes through a delayline of length four. Hence, the time it takes for a clock input signal CLK to reach to the register $j$ is $\sum_{i=0}^{3} \Delta t l_{0, i}+$ $\Delta t c_{j}$. Likewise, the time it takes for a data input signal to reach to a register $j$ is $\sum_{i=0}^{j<8} \Delta t l_{1, i}+\Delta t r_{j}$. Hence, we can derive and exploit the inequality of 5 . 

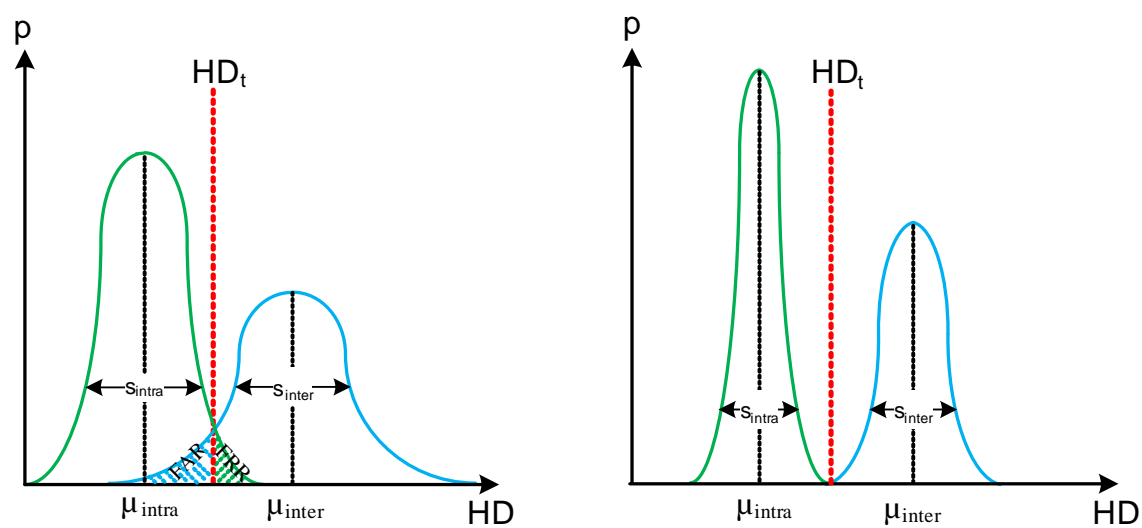

Figure 13. The effect of sampling on the distribution of HD [21]

$$
\sum_{i=0}^{j+1<8} \Delta t l_{1, i}+\Delta t r_{j+1} \geq \sum_{i=0}^{3} \Delta t l_{0, i}+t c_{j} \geq \sum_{i=0}^{j<8} \Delta t l_{1, i}+\Delta t r_{j}
$$

If the formula 5 is satisfied for any $0<j<8$, the value of $j^{\text {th }}$ register will become ' 1 ' while $j+1$ still remains at preset ' 0 '. Since $\Delta t l_{u, v}, t c_{i}$, and $\Delta t r_{i}$ are deviceunique random values, then the value of $j$, which is a linear combination of these values, will also be random. Note that, since we select the input trigger as the system clock, the skew between LUT1_0 and LUT0_0 is negligible as they are placed as adjacent slices.

Figure 12 shows the expected behaviour of a single PUF block. Depending on the delay variation between the delay-lines, the registers capture a thermometer code. For example, if DL1 is much faster than DL0, then the data will be ready on all registers before the clock edge and they will be set to '1' when the clock edge arrives. On the other hand, if DL1 is much slower than DL0, even the first register (REG_0) will receive its clock edge before the data input, all registers will miss the write ' 1 ' update and will remain in their preset value of ' 0 '. Due to process variation, the resulting response will be different on different FPGAs. Obviously, a single PUF block is not enough to realize an authentication solution for a large population. Hence, the system incorporates a number of these blocks to generate enough variance. The section 6 elaborates more on these issues.

The system integration process follows the methodology of section 4.2. The authentication system incorporates an MSP430 softcore processor [20]. The system integrates the MSP430 microprocessor with the PUF peripheral through a common memory-mapped interface. The system bus offers 16-bit transfers and thus can read two PUF block response simultaneously. Using a software in C programming language, the MSP430 can issue a PUF challenge, and collect and process the response.

\section{Results}

We have implemented the synchronous timingviolation based solution on the Altera Cyclone IV FPGAs and the asynchronous design on the Xilinx Virtex5 FPGAs. We have tested the quality of the generated authentication keys on 55 Altera and 100 Xilinx FPGAs. The synchronous design utilizes a NIOS-II softcore processor that runs at a constant $100 \mathrm{MHZ}$. We have also implemented the popular RO based PUF for comparison.

\subsection{Advanced Metrics of Quality for Authentication Identifiers}

The basic metrics of quality for the PUF constructions are single point estimates like uniqueness and reliability. However, as the main focus of this work is to provide physical authentication mechanism for SoCs, we provide advanced statistical estimations on the performance of authentication identifiers and the required block number to realize such results. In particular, based on our experiments, we will estimate the number of PUF blocks required to successfully authenticate a population size of a billion up to a trillion devices.

There are two metrics that relate to the authentication quality: false accept ratio (FAR) and false reject ratio (FRR). FAR refers to the counterfeits that happen to generate a valid ID and thus falsely identified as authentic. FRR, on the other hand, is the genuine entities 
Table 2. Statistical estimations of RO-based, Synchronous violation-based, and Asynchronous violation-based PUFs for authentication

\begin{tabular}{l|c|c|c|c|c|c|c|c} 
PUF architecture & $\mu_{\text {intra }}$ & $s_{\text {intra }}$ & $\mu_{\text {inter }}$ & $s_{\text {inter }}$ & $H D_{t\left(10^{-9}\right)}$ & $n_{\left(10^{-9}\right)}$ & $H D_{t\left(10^{-12}\right)}$ & $n_{\left(10^{-12}\right)}$ \\
\hline RO & 5.64 & 2.18 & 13.31 & 3.30 & 262 & 1857 & 345 & 2449 \\
RO-tmv & 2.00 & 1.54 & 13.70 & 2.10 & 36 & 324 & 47 & 428 \\
\hline Synchronous & 0.58 & 1.13 & 24.32 & 5.76 & 24 & 295 & 31 & 390 \\
Synchronous-tmv & 0.21 & 0.74 & 24.35 & 6.03 & 13 & 266 & 17 & 352 \\
\hline Asynchronous & 0.61 & 0.80 & 10.35 & 2.54 & 19 & 390 & 25 & 515 \\
Asynchronous-tmv & 0.24 & 0.50 & 10.33 & 2.52 & 10 & 304 & 13 & 402 \\
\hline
\end{tabular}

$H D_{t\left(10^{-9}\right)}$ : The hamming distance threshold to authenticate with an EER of $10^{-} 9$

$n_{\left(10^{-9}\right)}$ : The number of PUF blocks required to authenticate with an EER of $10^{-9}$

$H D_{t\left(10^{-12}\right)}$ : The hamming distance threshold to authenticate with an EER of $10^{-12}$

$n_{\left(10^{-12}\right)}$ : The number of PUF blocks required to authenticate with an EER of $10^{-12}$

mislabelled as fakes because of the PUF noise. Definitely, an authentication system aims to minimize both of these ratios.

Figure 13 visualizes the effect of increasing the number of PUF blocks on the intra and inter hamming distances. Central limit theorem guarantees that as the sample size increases, the standard deviation decreases while the mean remains the same. Therefore, as long as the mean intra hamming distance $\left(\mu_{\text {intra }}\right.$, noise $)$ is less than the mean inter hamming distance $\left(\mu_{\text {inter }}\right.$, process variation), deriving a reliable authentication mechanism is only an issue of gathering a large enough sample set per device by increasing the number of PUF blocks. Once we build such a system, we can set the threshold $H D_{t}$ to distinguish the authentic components from fakes. To compute the FAR and the FRR, we have modified the formulation of Bohm et al. [21]. The original definition assumes a gaussian distribution, however we have a moderate sample of a large population and we can not know the true standard deviation $(\sigma)$ of these populations. Hence, we applied a Student's t distribution that results in equations 6 and 7 as

$$
\begin{array}{r}
F R R=\int_{H D_{t}}^{\infty} \frac{\Gamma\left(\frac{v+1}{2}\right)}{\Gamma\left(\frac{v}{2}\right)} \frac{1}{\sqrt{v \pi}} \frac{1}{\left(1+\frac{t^{2}}{v}\right)^{\frac{v+1}{2}}} d x \times 100 \% \\
t=\frac{x-\mu_{\text {intra }}}{s_{\text {intra }} / \sqrt{n}} \\
F A R=\int_{-\infty}^{H D_{t}} \frac{\Gamma\left(\frac{v+1}{2}\right)}{\Gamma\left(\frac{v}{2}\right)} \frac{1}{\sqrt{v \pi}} \frac{1}{\left(1+\frac{t^{2}}{v}\right)^{\frac{v+1}{2}}} d x \times 100 \% \\
t=\frac{x-\mu_{\text {inter }}}{s_{\text {inter }} / \sqrt{n}}
\end{array}
$$

where, $\Gamma($.$) is the gamma function, t$ is the t-score, $n$ is the sample size, $v$ is the degree of freedom of the Student's t distribution that is set to $n-1$, and $s_{\text {intra }}$ and $s_{\text {inter }}$ are the point estimates to the intra and inter standard deviations, respectively. The results of the integral in equations 6 and 7 are the probability of a single observation from the Student's $t$ distribution to fall in the interval $\left[H D_{t}, \infty\right]$ and $\left[-\infty, H D_{t}\right]$. Note that for a large set, Student's t distribution becomes equivalent to the Gaussian distribution as the sample size $n \rightarrow \infty$.

Although $H D_{t}$ is useful to tradeoff the FAR and the FRR depending on the application requirements, we opt to choose it at the equal error rate (EER) where the FAR is equal to the FRR. Since $H D_{t}$ is a discrete value, we need to quantize the actual value of $H D_{t}$ to its nearest integer and compute the associated error rate. To achieve this, we follow the guidelines of [22] that formulates the error rates and the threshold as:

$$
H D_{t}=\operatorname{argmin}_{t}\left\{\max \left\{F A R\left(H D_{t}\right), F R R\left(H D_{t}\right)\right\}\right\}(8)
$$

$$
E E R=\max \left\{F A R\left(H D_{t}, F R R\left(H D_{t}\right)\right)\right\}
$$

We have implemented all PUF constructions with 64 instances generating 64-bits, and based on the observed output, we have estimated the required number of PUF blocks for authentication with an EER of $10^{-9}$ and $10^{-12}$. We also quantify the impact of temporal majority voting $(t m v)$ on the PUF responses. Table 2 gives the details of these experiments.

The synchronous design has a better authentication performance than the asynchronous design. The results indicate that the primary reason for this is not the reliability of the PUFs but the difficulty of extracting deviceunique variation on Xilinx FPGAs with the proposed design. Indeed, the mean hamming distance of the synchronous design is quite larger than the rest of the candidates. As expected, RO have the worst performance 
due to the placement and routing limitation of the Altera toolchain. RO are much unreliable than the timing violation-based solutions. Although the reliability improves significantly with the temporal majority voting, it requires repeating PUF measurements which is, compared to our solutions, takes much longer due to the measurement time of the oscillators. Recent work also complies with our results and shows that on similar Altera FPGAs, the quality of RO based PUFs can change drastically and can have an anomalous characteristics [23]. However, the typical quality metrics for RO-PUFs on Xilinx FPGAs are much better than what is achieved on Altera FPGAs [13].

The cost of a single synchronous PUF block is 32 registers to store the output data, and 48 LUTs. Therefore, it can be placed into $3 \mathrm{LABs}$. On the other hand, one RO-PUF requires just $1 \mathrm{LAB}$ resulting in a smaller area per PUF block. The asynchronous PUF block costs 12 LUTs and 8 registers and thus can fit into only 3 slices.

\section{Conclusions}

In this paper, we have described a complete solution to physically authenticate stable-clocked SoC platforms. The system utilizes a novel PUF construction that enables a seamless integration to its SoC architecture. The main improvement of the proposed PUFs over the previous work [5] is being able to operate at a stable-clock operation. The SoC integration is performed using the custom instruction interface of the NIOS-II processor and the memory mapped interface of the MSP430 processor. Hence, PUF responses can be issued at run-time using instruction calls from the processor. This brings the flexibility of the software postprocessing and allows a practical physical authentication of the platform.

The proposed PUFs are time-to-digital converters that consist of delay-lines. These architectures allow determining the critical timing violation path which has static randomness due to manufacturing process variations. We have implemented our prototypes on Altera Cyclone IV and Xilinx Virtex-5 FPGAs and have tested the physical authentication method on 55 and 100 boards, respectively. The proposed solution outperforms other timing violation based PUFs [5] in terms of the quality of the generated responses. Moreover, we show that our solutions are more efficient than the ring oscillator based constructions. Since the PUF block is architecturally visible by the SoC, the future work could be realizing full security protocols on the platform and using the proposed PUF structure as a basic building block.
For the experimental setup we have used 64 copies of the PUF blocks and estimated the required number of copies to succesfully authenticate a large population of devices. We showed that depending on the security requirements of the application domain (from minimalsecurity to military-grade), the proposed solution is easily scalable by incorporating more PUF blocks and can generate authentication identifiers of various size.

\section{Acknowledgements}

This work was supported in part through National Science Foundation Grant 0964680. We also want to acknowledge Ege Gulcan and Bilgiday Yuce for their contributions to the measurements.

\section{References}

[1] S. Devadas, E. Suh, S. Paral, R. Sowell, T. Ziola, V. Khandelwal, Design and implementation of PUF-based "unclonable" RFID ICs for anti-counterfeiting and security applications, in: RFID, 2008 IEEE International Conference on, 2008, pp. 5864. doi:10.1109/RFID.2008.4519377.

[2] K. Kursawe, A. Sadeghi, D. Schellekens, B. Skoric, P. Tuyls, Reconfigurable physical unclonable functions - enabling technology for tamper-resistant storage, in: Hardware-Oriented Security and Trust, 2009. HOST '09. IEEE International Workshop on, 2009, pp. 22-29. doi:10.1109/HST.2009.5225058.

[3] J. Guajardo, S. Kumar, G.-J. Schrijen, P. Tuyls, Physical unclonable functions and public-key crypto for FPGA IP protection, in: Field Programmable Logic and Applications, 2007. FPL 2007. International Conference on, 2007, pp. 189-195. doi:10.1109/FPL.2007.4380646.

[4] M. Gora, A. Maiti, P. Schaumont, A flexible design flow for software IP binding in FPGA, Industrial Informatics, IEEE Transactions on 6 (4) (2010) 719-728. doi:10.1109/TII.2010.2068303.

[5] A. Maiti, P. Schaumont, A novel microprocessor-intrinsic physical unclonable function, in: Field Programmable Logic and Applications (FPL), 2012 22nd International Conference on, 2012, pp. 380-387. doi:10.1109/FPL.2012.6339208.

[6] J. S. J. Wong, P. Sedcole, P. Y. K. Cheung, Self-measurement of combinatorial circuit delays in FPGAs, ACM Trans. Reconfigurable Technol. Syst. 2 (2) (2009) 10:1-10:22. doi:10.1145/1534916.1534920.

URL http: //doi . acm.org/10.1145/1534916.1534920

[7] Y. Hori, T. Yoshida, T. Katashita, A. Satoh, Quantitative and statistical performance evaluation of arbiter physical unclonable functions on FPGAs, in: Reconfigurable Computing and FPGAs (ReConFig), 2010 International Conference on, 2010, pp. 298303. doi:10.1109/ReConFig.2010.24.

[8] M. Majzoobi, F. Koushanfar, S. Devadas, FPGA PUF using programmable delay lines, in: Information Forensics and Security (WIFS), 2010 IEEE International Workshop on, 2010, pp. 1-6. doi:10.1109/WIFS.2010.5711471.

[9] J. Anderson, A PUF design for secure FPGA-based embedded systems, in: Design Automation Conference (ASPDAC), 2010 15th Asia and South Pacific, 2010, pp. 1-6. doi:10.1109/ASPDAC.2010.5419927.

[10] R. Maes, P. Tuyls, I. Verbauwhede, Intrinsic PUFs from Flipflops on Reconfigurable Devices, in: Benelux Workshop on Information and System Security, 2008. 
[11] T. Güneysu, Using data contention in dual-ported memories for security applications, Journal of Signal Processing Systems 67 (1) (2012) 15-29. doi:10.1007/s11265-010-0560-z. URL http://dx.doi .org/10.1007/s11265-010-0560-z

[12] S. Kumar, J. Guajardo, R. Maes, G.-J. Schrijen, P. Tuyls, Extended abstract: The butterfly PUF protecting IP on every FPGA, in: Hardware-Oriented Security and Trust, 2008. HOST 2008. IEEE International Workshop on, 2008, pp. 6770. doi:10.1109/HST.2008.4559053.

[13] A. Maiti, P. Schaumont, Improving the quality of a physical unclonable function using configurable ring oscillators, in: Field Programmable Logic and Applications, 2009. FPL 2009. International Conference on, 2009, pp. 703-707. doi:10.1109/FPL.2009.5272361

[14] J. Aarestad, P. Ortiz, D. Acharyya, J. Plusquellic, HELP: A hardware-embedded delay PUF, Design Test, IEEE 30 (2) (2013) 17-25. doi:10.1109/MDT.2013.2247459.

[15] R. Maes, I. Verbauwhede, Physically unclonable functions: A study on the state of the art and future research directions, in: A.-R. Sadeghi, D. Naccache (Eds.), Towards HardwareIntrinsic Security, Information Security and Cryptography, Springer Berlin Heidelberg, 2010, pp. 3-37. doi:10.1007/9783-642-14452-3_1.

URL http: //dx .doi .org/10.1007/978-3-642-14452-3_1

[16] Z. Cherif, J.-L. Danger, S. Guilley, L. Bossuet, An easy-todesign PUF based on a single oscillator: The loop PUF, in: Digital System Design (DSD), 2012 15th Euromicro Conference on, 2012, pp. 156-162. doi:10.1109/DSD.2012.22.

[17] Altera Corperation, NIOS II custom instruction user guide, Tech. rep. (January 2011)

[18] J. Ju, R. Chakraborty, C. Lamech, J. Plusquellic, Stability analysis of a physical unclonable function based on metal resistance variations, in: Hardware-Oriented Security and Trust (HOST), 2013 IEEE International Symposium on, 2013, pp. 143-150. doi:10.1109/HST.2013.6581580.

[19] G. Suh, S. Devadas, Physical unclonable functions for device authentication and secret key generation, in: Design Automation Conference, 2007. DAC '07. 44th ACM/IEEE, 2007, pp. 9-14.

[20] O. Girard, openmsp430 (2009). URL http: //opencores . org/project, openmsp430

[21] C. Böhm, M. Hofer, Testing and specification of pufs, in: Physical Unclonable Functions in Theory and Practice, Springer New York, 2013, pp. 69-86. doi:10.1007/978-1-4614-5040-5_4. URL http: //dx . doi .org/10.1007/978-1-4614-5040-5_4

[22] R. Maes, PUF-based entity identification and authentication, in: Physically Unclonable Functions, Springer Berlin Heidelberg, 2013, pp. 117-141. doi:10.1007/978-3-642-41395-7_5. URL http: //dx .doi .org/10.1007/978-3-642-41395-7_5

[23] L. Feiten, A. Spilla, M. Sauer, T. Schubert, B. Becker, Analysis of ring oscillator PUFs on 60nm FPGAs.

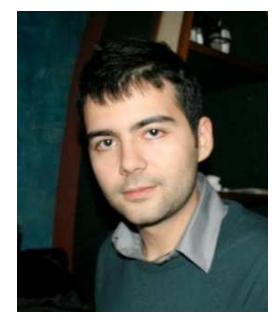

Aydin Aysu received his B.S. degree in Microelectronics and his M.S degree in Electronics Engineering from Sabanci University, Istanbul, Turkey, in 2008 and 2010, respectively. He worked as a digital design engineer at Vestek R\&D Co. until 2012. He is currently working towards his Ph.D. degree at the Department of Electrical and Computer Engineering, Virginia Tech, Blacksburg. His research interests include embedded system security and secure hardware design.

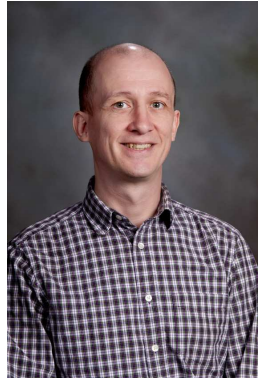

Patrick Schaumont is an Associate Professor in Computer Engineering at Virginia Tech. He received the $\mathrm{PhD}$ degree in Electrical Engineering from UCLA (2004). His research addresses the major challenges in cryptographic engineering, and the conception, design, and implementation of nextgeneration embedded systems. He has served on the program committee of international conferences in this field such as CHES, DATE, DAC, IEEE HOST. He is associate editor with several journals in this field including IEEE TC, IACR JCEN, ACM TODAES and ACM TECS. He is a senior member of the IEEE and the member of the IACR. 